(C) 2010 IEEE. Reprinted, with permission, from Cardellach, E., Rius, A., Cerezo, F., Garcia-Primo, M.A., de la Torre-Juarez, M., Cucurull, L., Ector, D., Polarimetric GNSS Radio-Occultations for heavy rain detection, Proceedings of IEEE International Geoscience and Remote Sensing Symposium (IEEE IGARSS), and july/2010.

This material is posted here with permission of the IEEE. Such permission of the IEEE does not in any way imply IEEE endorsement of any of CSIC products or services. Internal or personal use of this material is permitted. However, permission to reprint/republish this material for advertising or promotional purposes or for creating new collective works for resale or redistribution must be obtained from the IEEE by writing to pubs-permissions@ieee.org.

By choosing to view this document, you agree to all provisions of the copyright laws protecting it. 


\title{
POLARIMETRIC GNSS RADIO-OCCULTATIONS FOR HEAVY RAIN DETECTION
}

\author{
E. Cardellach ${ }^{1}$, A. Rius ${ }^{1}$, F. Cerezo $^{2}$, M.A. García-Primo ${ }^{2}$, M. de la Torre-Juárez ${ }^{3}$, L. Cucurull $^{4}$, D. Ector ${ }^{4}$ \\ ${ }^{1}$ Institut de Ciències de l'Espai, ICE/CSIC-IEEC, Barcelona, Spain \\ ${ }^{2}$ HISDESAT, Madrid, Spain \\ ${ }^{3}$ Jet Propulsion Laboratory, California Institute of Technology, Pasadena, CA, USA \\ ${ }^{4}$ NOAA, Washington D.C, USA
}

\begin{abstract}
A Global Navigation Satellite System (GNSS) Radio-Occultation (RO) experiment is being accommodated in the Spanish Low Earth Orbiter (LEO) for Earth Observation PAZ. The ROpayload will provide globally distributed vertical thermodynamic profiles of the Atmosphere in Near Real-Time (NRT) suitable to be assimilated into Weather Numerical Prediction Models. The NRT operability will be dispensed by the Ground Segment services of the U.S.A. National Oceanographic and Atmospheric Administration (NOAA). Moreover, the mission will run, for the first time from Space, a double-polarization GNSS experiment, to assess the capabilities of polarimetric GNSS-RO for detection of heavy rain events.
\end{abstract}

Index Terms - GNSS, GNSS Remote Sensing, GNSS Radio-Occultations, Atmospheric Profiles, Heavy Precipitation

\section{INTRODUCTION}

The Spanish Ministry for Science and Innovation (MICINN) has recently approved a proposal to include a polarimetric Global Navigation Satellite System (GNSS) Radio-Occultation (RO) payload on board of the Spanish Earth Observation satellite PAZ. The PAZ mission, planned to be launched in Spring 2012, was initially designed to carry a Synthetic Radar Aperture (SAR) as primary and sole remote sensing payload, and it included an IGOR+ advanced Global Positioning System (GPS) receiver and corresponding antennas for precise orbit determination. After minor modifications, the design and software of this GPS receiver will allow the tracking of

ROHPP is funded by the Spanish Ministry of Science and Innovation, projects ACI2009-1023 and AYA2008-05906-C02-02/ESP. E.C. is funded by the Ramón y Cajal Programme. The ground campaign antenna is provided by the UPC. Part of this research is carried out at the Jet Propulsion Laboratory, California Institute of Technology, under a contract with the National Aeronautics and Space Administration. Members of the ROHPP International Science Team have contributed to the scientific requirements of the ROHPP, ROHPP proposal, and the ground campaign definition (particuarly to J. Turk, R. Kursinski, and T. Meehan). Figure 5 was generated with data provided by CLABSA.

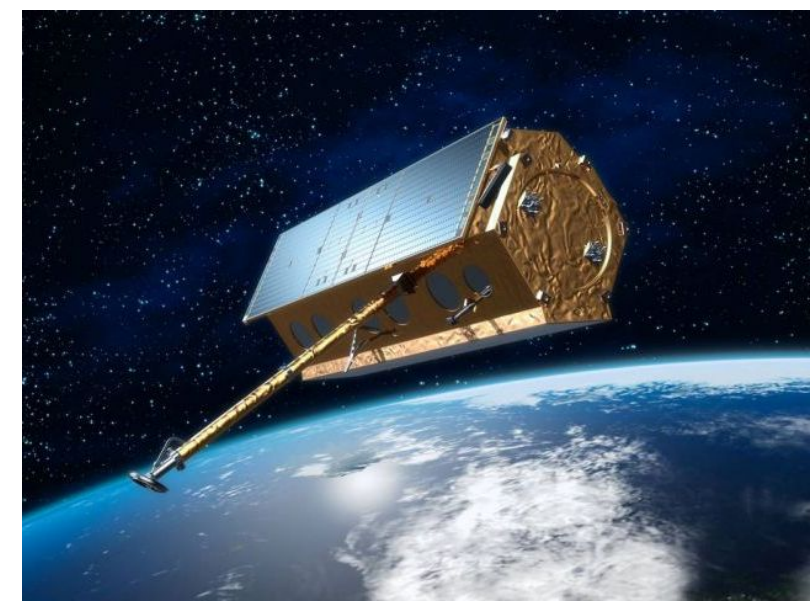

Fig. 1. Artistic view of PAZ satellite.

occulting signals, that is: signals transmitted by GPS satellites setting below the horizon of the Earth (or rising above it). The Radio Occultation technique originated in Planetary sciences for the study of other Planets' atmospheres, and measures the delay caused by the atmospheric refractivity gradients on the propagation of the radio link. This delay can be used to infer vertical radio-refractivity profiles from which ionospheric total electron content can be inferred as well as vertical profiles of thermodynamic variables, such as atmospheric pressure, temperature, water vapor pressure, from the stratosphere down to the boundary layer with a vertical resolution close to $300 \mathrm{~m}$. RO thermodynamical profiles are assimilated operationally into several global numerical weather prediction models (NWPM) [1]. Latest results at NCEP show that ROs improve anomaly correlation scores by $\sim 8 \mathrm{hr}$ starting at day 4 and increases with extended forecast range. It also helps reducing model biases. ECMWF compared the impact of 24 operational observation systems, GPS-RO impact resulting among the top-five [2]. The use of GPS RO has been shown to significantly improve models forecast skill, and is a key component of the operational observing system [3]. 
Table 1. Table of missions carrying a RO experiment and their period of coverage.

\begin{tabular}{llll} 
RO Mission & $\begin{array}{l}\text { Expected } \\
\text { Start }\end{array}$ & $\begin{array}{l}\text { Expected } \\
\text { end of Life }\end{array}$ & Finished \\
\hline GPS/MET & $04 / 03 / 95$ & $03 / 17 / 97$ & $3 / 17 / 97$ \\
SAC-C & $10 / 05 / 01$ & $05 / 10 / 06$ & N/A \\
CHAMP & $02 / 01 / 01$ & $02 / 01 / 06$ & $10 / 15 / 08$ \\
GRACE & $07 / 15 / 04$ & $03 / 10 / 07$ & N/A \\
COSMIC(6) & $05 / 01 / 06$ & $05 / 01 / 11$ & N/A \\
GRAS/METOP & $10 / 27 / 06$ & $10 / 27 / 11$ & N/A \\
TerraSAR-X & $06 / 05 / 07$ & $07 / 01 / 12$ & N/A \\
OCEANSAT-2 & $09 / 23 / 09$ & $09 / 23 / 14$ & N/A \\
SAC- & 2010 & 2015 & N/A \\
D/Aquarius & & & \\
Kompsat & 2010 & 2015 & N/A \\
PAZ & 2012 & 2017 & N/A \\
\hline
\end{tabular}

Missions currently providing this information to the NWPM are the constellation of 6 Low-Earth Orbiters (Taiwan/USA FORMOSAT-3/COSMIC mission), and the EUMETSAT's GRAS instrument aboard the METOP satellite, in the form of globally distributed profiles. However, the number of RO profiles risks to dramatically drop after the decommissioning of the COSMIC constellation. With a mission life-expectancy of 5 years, the current yield of COSMIC is not ensured after 2011. PAZ is planned to be launched when this happens and before a possible new RO constellation is deployed (see Table 1 for the calendar of RO missions, note that only some of them can provide RO data in NRT for operational ingestion into NWPM).

\section{PRECIPITATION EXPERIMENT}

The GPS works at L-band (e.g. L1-GPS $\sim 1.5 \mathrm{GHz}$ ) and Right-Hand Circular Polarization RHCP). Although the frequencies of these signals were chosen such that they would suffer low attenuation by clouds or rain and enable allweather operations, the de-polarization effect induced by the flattening of the heavy precipitation drops may exceed some measurable threshold. Work done for calibration and characterization of similar signals (GOES 11 L-band down-link at $1.5445 \mathrm{GHz}$ RHCP) have found strong correlation between the de-polarization ratio and heavy rain effects, when measuring a down-link at $14^{\circ}$ elevation angle (above the horizon) [4]. The same concept, in backscattering geometry rather than propagation, is used in the NEXRAD network of polarimetric weather radars across U.S.A, working at $3 \mathrm{GHz}$ (twice L1-GPS). The de-polarization effect increases as the propagation line aligns with the plane of the drops' flattening (usually parallel to the local horizon). The Radio-Occultation signals cross the lower troposphere tangentially, i.e. along the local horizon, which maximize the de-polarization effect.

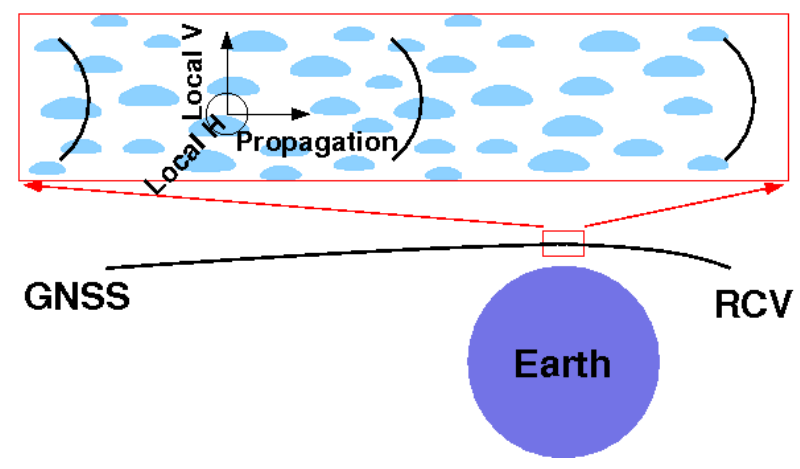

Fig. 2. Sketch of the RO-Heavy Precipitation measurement concept: at lower troposphere, the RHCP GPS signal propagates tangentially, aligned with the plane of flatness of the rain drops, situation in which the de-polarization effect is maximal. The receiver on board the PAZ mission will collect both polarization components.

The concept is sketched in Figure 2.

PAZ will include RO capabilities with a new approach for the relatively small limb-oriented GPS antennas. The proposed RO antenna will use the GPS L-band RHCP transmitted signals to capture both co- and cross-polarized components of the GPS propagated signal for the first time in a space-based GPS receiver. This experiment will be a proof-of-concept to exploit the potential capabilities of polarimetric radio occultation towards detecting and quantifying heavy precipitation events. If successful, PAZ will open new applications of GNSS Radio-Occultation observations by providing coincident thermodynamic and precipitation information with high vertical resolution within regions covered by thick clouds.

Precipitation remains a poorly predicted event with current climate and weather model parameterizations. A better understanding of the thermodynamics of heavy precipitation events is necessary towards improving climate models and quantifying the impact of climate variability on precipitation $[5,6]$. Just flagging the existence of precipitation within RO refractivity profiles will help a better understanding of the thermodynamic conditions underlying precipitation. The particular advantage of GNSS RO is that their signals are in the microwave spectrum which is influenced little by clouds.

A few infrared sensors, such as AIRS, can profile atmospheric temperature/humidity with high vertical resolution but cannot penetrate through the thick clouds typically associated with heavy precipitation.

The PAZ mission will partially overlap with the core instrument of the Global Precipitation Mission (GPM), to be launched in 2013. The timing of the PAZ RO-Heavy Precipitation concept will enable to compare RO coincident thermodynamical and precipitation information with the GPM mission. This synergy might also make PAZ a potential candidate to complement GPM by measuring additional aspects of pre- 


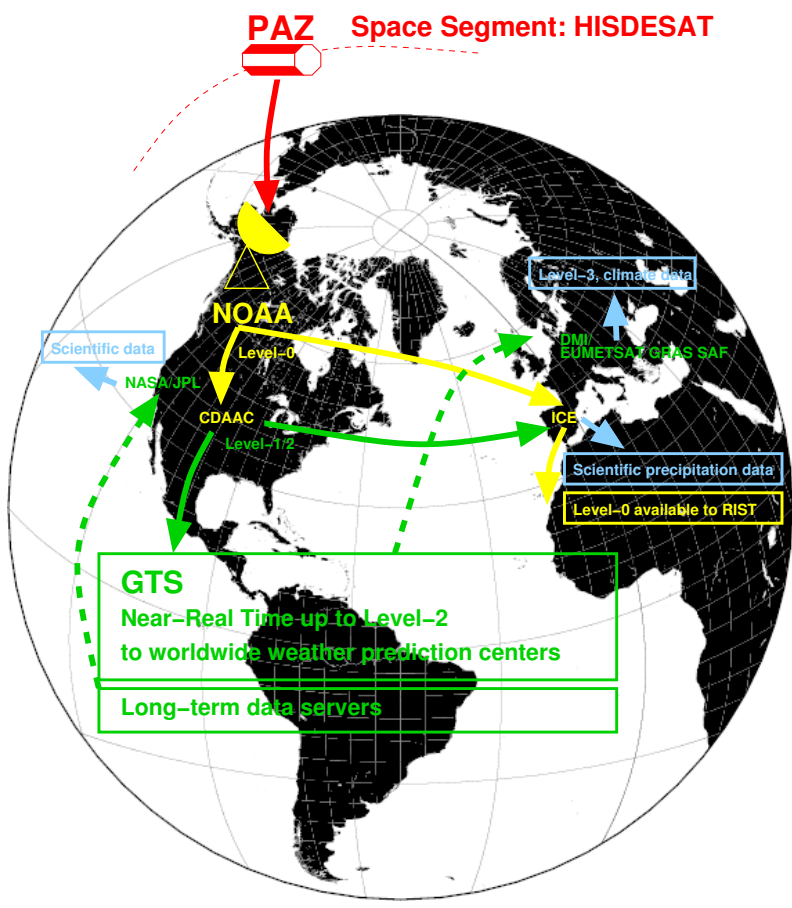

Fig. 3. Sketch of the ROHPP ground-segment.

cipitation processes. PAZ orbit is near-polar, which ensures dense coverage over high latitudes, one of the requirements of the GPM.

\section{SPACE AND GROUND SEGMENTS}

ICE-CSIC/IEEC leads the Radio-Occultation and Heavy Precipitation with PAZ (ROHPP) project, to bring polarimetric RO capabilities into the PAZ platform. HISDESAT is the company in charge of the implementation of the overall PAZ mission, including the operation and ground segment of the main, SAR, payload. Because of the original PAZ-SAR telemetry ground-station location, the data will be downlinked once per day, beyond NRT time-line. An alternative ground segment system has been found to permit NRT meteorological operational use of the ROHPP data. HISDESAT will operate the Space Segment, and it will telemeter the ROHPP data to a NOAA suitable ground-station once per orbit. NOAA's ground-segment facilities and strategy deployed for the COSMIC RO mission will be re-used for ROHPP, with the aim of delivering PAZ atmospheric profiles to the Global Telecommunications System (GTS) with less than 2 hour latency. This will involve the COSMIC Data Analysis and Archive Center (CDAAC). ICE-CSIC/IEEC will maintain an alternative data server, including raw data for conducting research on polarimetric capabilities.
Table 2. Calendar of the PAZ mission.

\begin{tabular}{ll} 
Calendar Milestone & $\begin{array}{l}\text { Dates } \\
\text { (dd/mm/yy) }\end{array}$ \\
\hline Platform Critical Design Review (CDR) & $15 / 10 / 09$ \\
CDR & $15 / 12 / 09$ \\
Platform Test readiness review (TRR) & $16 / 08 / 10$ \\
Platform Delivery Review Board (DRB) & $24 / 12 / 10$ \\
GPS receiver (IGOR) delivery & $31 / 01 / 11$ \\
Satellite Integration starts & $28 / 02 / 11$ \\
Satellite Test Readiness Review & June 2011 \\
Final Acceptance Review (FAR) & $19 / 12 / 11$ \\
Launch Readiness Review (LRR) & $19 / 01 / 12$ \\
\hline
\end{tabular}

\section{CURRENT STATUS AND RESEARCH}

The Critical Design Review (CDR) of the entire mission has been passed in December 2009, and the RO payload is being manufactured. The RO related payload is expected to be delivered for integration into the platform by January 2011. The satellite integration should start during February 2011, to pass the test readiness (June 2011); acceptance (December 2011); and launch readiness (January 2012) reviews on time (see calendar in Table 2).

Meanwhile, the ROHPP International Science Team (RIST) has been defined, involving scientists from ICECSIC/IEEC; NOAA; NASA/Jet Propulsion Laboratory (JPL); U. Arizona; EUMETSAT's GRAS SAF (Danish Meteorological Institute-DMI-and European Centre for Medium Weather Forecast-ECMWF); German Research Centre for Geo-sciences (GFZ); U. Castilla la Mancha (UCLM); and Polytechnic U. of Catalonia (UPC). A ground campaign is being prepared, as well as other studies in support the PAZ mission concept.

The ground campaign will take place in an elevated site near Barcelona (Spain), at more than 1700 meter altitude. The site has clear views over the horizon, especially towards the South, where many GPS signals rise/set (Figure 4). The area of observation suffers several short heavy and violent rain events along the year (Figure 5). The GPS Open-Loop Differential Real-Time Receiver (GOLD-RTR [7]) will be used, together with a steerable high-gain double-polarization $(\mathrm{V} / \mathrm{H})$ GPS antenna being developed at the UPC. The goal of the campaign is to characterize the polarimetric effects in the GPS tangentially propagating signals, as well as to identify systematic effects that might affect the PAZ space-borne observations. In order to achieve these objectives, the equipment will be constantly monitoring the horizon for a period of two to three months.

Analysis of non-polarimetric COSMIC RO is been performed to evaluate the possibility that de-polarization effects could be identified via attenuation measurements of the RO signal, but it is unclear that they can be detected yet. In parallel, we are applying the theory of radio-wave propagation 


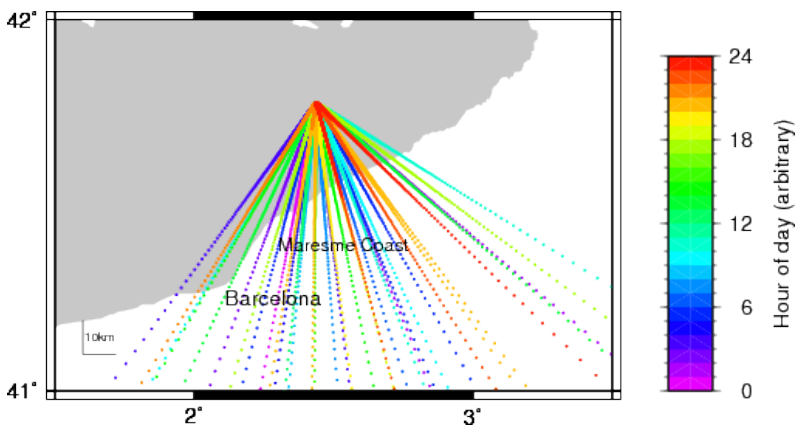

Fig. 4. Approximate Geo-location of the points in which the rays connecting the GPS transmitters and the receiver cross the 3000 meter altitude layer (no bending considered). An azimuthal mask has been applied to account for the area with clear visibility over the horizon. The color scale represent the hour of the day in which the observation happens $(\sim 4$ minutes daily shift).

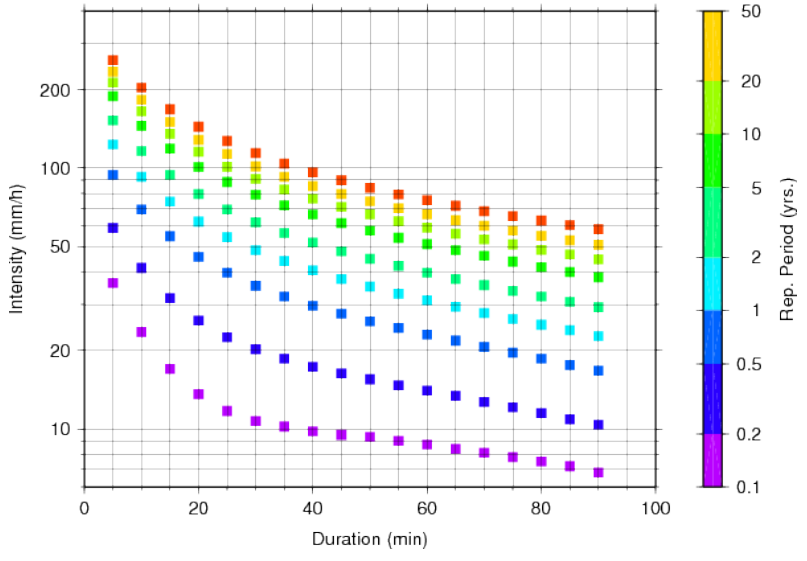

Fig. 5. Duration-Rate-Repeatability (DRR) chart corresponding to a single station in Barcelona city, computed from 66 years of accumulated records. through a precipitating medium at the GPS frequencies [8, 9] to predict the rain rates to which PAZ can be sensitive. Preliminary estimates suggest that the PAZ RO experiment will require strong de-polarization effects associated to very heavy precipitation events along extended RO ray-paths. Work remains to quantify the sensitivity more precisely, The outcome of the experiment will help defining the real sensitivity of the concept, together with requirements for the optimal design of future precipitation-RO instruments.

\section{REFERENCES}

[1] S.B. Healy, A.M. Jupp, and C. Marquardt, "Forecast impact experiment with GPS radio occultation measurements," Geophys. Res. Lett., vol. 32, 2005.

[2] C. Cardinali, "Forecast sensitivity to observation (FSO) as a diagnostic tool," Tech. Rep., ECMWF Technical Memorandum 599, Reading, UK, 2009.

[3] NRC Committee on Earth Science and Applications from Space, "A community assessment and strategy for the future, earth science and applications from space, national imperatives for the next decade and beyond," Tech. Rep., The National Academies Press, Washington DC, USA, 2007.

[4] R. Peters, P. Woolner, and E. Ekelman, "Analytic calculation of noise power robbing, NRP, and polarization isolation degradation," in Proceedings of 26th International Communications Satellite Systems Conference (ICSSC). AIAA, June 2008.

[5] F.J. Wentz, L. Ricciardulli, K. Hilburn, and C. Mears, "How much more rain will global warming bring?," Science, vol. 317, no. 5835, 2007.

[6] R.P. Allan and B.J. Soden, "Atmospheric warming and the amplification of precipitation extremes," Science, vol. 321, no. 5895, pp. 1481-1484, 2008.

[7] O. Nogués-Correig, E. Cardellach Galí, J. Sanz Campderròs, and A. Rius, "A GPS-Reflections Receiver That Computes Doppler/Delay Maps in Real Time," vol. 45, no. 1, pp. 156-174, Jan. 2007.

[8] T. S. Chu, "Microwave depolarization of an earth-space path,” Bell Syst. Tech. J., vol. 59, pp. 987-1007, 1980.

[9] W.L. Flock, "Propagation effects on satellite systems for frequencies below $10 \mathrm{GHz}$," NASA Ref. Publ., vol. 1108, no. 2 . 